\title{
AN INEQUALITY FOR CAUCHY PRODUCTS
}

\author{
JAVAD MASHREGHI
}

(Communicated by Stephan Ramon Garcia)

Abstract. We show that $\ell^{p}$ is closed under the Cauchy product by $\ell^{1}$ sequences.

\section{INTRODUCTION}

Let $f$ and $g$ be two analytic functions in a neighborhood of origin with Taylor series expansions

$$
f(z)=\sum_{n=0}^{\infty} a_{n} z^{n} \quad \text { and } \quad g(z)=\sum_{n=0}^{\infty} b_{n} z^{n} .
$$

The usual product of $f$ and $g$ has Taylor series expansion

$$
(f g)(z)=\sum_{n=0}^{\infty} c_{n} z^{n}
$$

where the coefficients $c_{n}$ are given by

$$
c_{n}=\sum_{k=0}^{n} a_{k} b_{n-k} \quad(n \geq 0) .
$$

The sequence $\left(c_{n}\right)_{n \geq 0}$ is called the Cauchy product of $\left(a_{n}\right)_{n \geq 0}$ and $\left(b_{n}\right)_{n \geq 0}$,2, pp. 227-229]. It may also be regarded as the discrete convolution of $\left(a_{n}\right)_{n \geq 0}$ and $\left(b_{n}\right)_{n \geq 0}$ [3, pp. 13-20].

There are several questions that arise in connection with Cauchy products of infinite sequences when we study the multiplier algebra of Banach spaces of analytic functions on the open unit disc $\mathbb{D}$. The characterization of the multiplier algebra itself, or at least a subalgebra of it, is one of the main issues. In this situation, we restrict $f$ to a Banach space of analytic functions and ask for which $g$ the image $g f$ stays in the space. No doubt, there is no universal answer, and the final characterization depends on the geometry of the Banach space under consideration. In this short note, we just consider the classical $\ell^{p}$ space with $1<p<\infty$. The corresponding Banach space of analytic functions on $\mathbb{D}$ is denoted by $\ell_{A}^{p}[1]$. The characterization of the multiplier algebra of $\ell^{p}$, or equivalently of $\ell_{A}^{p}$, is still an open question. We show that $\ell^{1}$ continuously (even contractively) embeds in the

Received by the editors May 3, 2016 and, in revised form, August 15, 2016.

2010 Mathematics Subject Classification. Primary 47A30; Secondary 26D15, 47A63.

This work was supported by NSERC (Canada). 
multiplier algebra of $\ell^{p}$. More explicitly, we study sequences $\left(a_{n}\right)_{n \geq 0}$ for which

$$
\sum_{n=0}^{\infty}\left|\sum_{k=0}^{n} a_{k} b_{n-k}\right|^{p}<\infty
$$

for all $\left(b_{n}\right)_{n \geq 0} \in \ell^{p}$. Clearly, this holds whenever $\left(a_{n}\right)_{n \geq 0}$ is a compactly supported sequence, or equivalently when $f$ is a polynomial. But, we provide a quantitative evaluation of (1.2) which works for a wide class of infinite sequences.

\section{MAIN RESUlT}

Without loss of generality, we assume that all coefficients are positive. The passage to the more general setting of complex numbers is straightforward.

Theorem 2.1. Let $\left(a_{n}\right)_{n \geq 0}$ and $\left(b_{n}\right)_{n \geq 0}$ be two sequences of positive numbers. Let $1<p<\infty$, with exponent conjugate $q$, and let $0<r<1$. Then

$$
\left(\sum_{n=0}^{\infty}\left(\sum_{k=0}^{n} a_{k} b_{n-k}\right)^{p}\right)^{\frac{1}{p}} \leq\left\{\left(\sum_{n=0}^{\infty} a_{n}^{r q}\right)^{\frac{1}{q}}\left(\sum_{n=0}^{\infty} a_{n}^{(1-r) p}\right)^{\frac{1}{p}}\right\}\left(\sum_{n=0}^{\infty} b_{n}^{p}\right)^{\frac{1}{p}} .
$$

Proof. The proof is based on a judicial application of the classical Hölder inequality. Write $a_{k} b_{n-k}=a_{k}^{r} \cdot\left(a_{k}^{1-r} b_{n-k}\right)$. Then, by Hölder's inequality,

$$
\sum_{k=0}^{n} a_{k} b_{n-k} \leq\left(\sum_{k=0}^{n} a_{k}^{r q}\right)^{\frac{1}{q}}\left(\sum_{k=0}^{n} a_{k}^{(1-r) p} b_{n-k}^{p}\right)^{\frac{1}{p}} \quad(n \geq 0) .
$$

Hence,

$$
\left(\sum_{k=0}^{n} a_{k} b_{n-k}\right)^{p} \leq\left(\sum_{k=0}^{\infty} a_{k}^{r q}\right)^{\frac{p}{q}}\left(\sum_{k=0}^{n} a_{k}^{(1-r) p} b_{n-k}^{p}\right) \quad(n \geq 0) .
$$

Note that in the second summation, we replaced $n$ by $\infty$. Now, we add all these inequalities and change the order of summation on the right side:

$$
\begin{aligned}
\sum_{n=0}^{\infty}\left(\sum_{k=0}^{n} a_{k} b_{n-k}\right)^{p} & \leq\left(\sum_{k=0}^{\infty} a_{k}^{r q}\right)^{\frac{p}{q}} \times \sum_{n=0}^{\infty}\left(\sum_{k=0}^{n} a_{k}^{(1-r) p} b_{n-k}^{p}\right) \\
& \leq\left(\sum_{k=0}^{\infty} a_{k}^{r q}\right)^{\frac{p}{q}} \times \sum_{k=0}^{\infty}\left(\sum_{n=k}^{\infty} a_{k}^{(1-r) p} b_{n-k}^{p}\right) \\
& \leq\left(\sum_{k=0}^{\infty} a_{k}^{r q}\right)^{\frac{p}{q}} \times \sum_{k=0}^{\infty} a_{k}^{(1-r) p} \times \sum_{n=0}^{\infty} b_{n}^{p} .
\end{aligned}
$$

Finally, take the $p$-th root of both sides.

The parameter $r$ is free to run between 0 and 1. However, at the point of intersection of lines $r \longmapsto r q$ and $r \longmapsto(1-r) p$, i.e., for $r=p /(p+q)$, something interesting happens.

Corollary 2.2. Let $\left(a_{n}\right)_{n \geq 0}$ and $\left(b_{n}\right)_{n \geq 0}$ be two sequences of positive numbers. Let $1<p<\infty$. Then

$$
\left(\sum_{n=0}^{\infty}\left(\sum_{k=0}^{n} a_{k} b_{n-k}\right)^{p}\right)^{\frac{1}{p}} \leq\left(\sum_{n=0}^{\infty} a_{n}\right)\left(\sum_{n=0}^{\infty} b_{n}^{p}\right)^{\frac{1}{p}}
$$


Briefly speaking, Corollary 2.2 says that the Cauchy product of a sequence in $\ell^{1}$ with another in $\ell^{p}$ stays in $\ell^{p}$.

\section{Equality}

Let us study the case of equality in Corollary 2.2. The procedure is similar for the other inequality in Theorem 2.1. We show that equality holds just in the following cases:

(i) $\left(a_{n}\right) \equiv 0$ (trivial case).

(ii) $\left(b_{n}\right) \equiv 0$ (trivial case).

(iii) There is an integer $k$ such that $a_{n}=0$ for all $n \geq 0$ except possibly for $n=k$ (interesting case).

The verification of the third case is a bit delicate and needs special care.

At two stages, we have an inequality in the proof of Corollary 2.2 which must turn into an equality. First, equality holds in

$$
\sum_{k=0}^{n} a_{k} b_{n-k} \leq\left(\sum_{k=0}^{n} a_{k}\right)^{\frac{1}{q}}\left(\sum_{k=0}^{n} a_{k} b_{n-k}^{p}\right)^{\frac{1}{p}} \quad(n \geq 0)
$$

if and only if one of the following two cases happens:

(i) $\left(a_{0}^{1 / q}, a_{1}^{1 / q}, \ldots, a_{n}^{1 / q}\right)=0$ or $\left(a_{0}^{1 / p} b_{n}, a_{1}^{1 / p} b_{n-1}, \ldots, a_{n}^{1 / p} b_{0}\right)=0$.

(ii) $\left(a_{0}^{1 / q}, a_{1}^{1 / q}, \ldots, a_{n}^{1 / q}\right) \neq 0$ and $\left(a_{0}^{1 / p} b_{n}, a_{1}^{1 / p} b_{n-1}, \ldots, a_{n}^{1 / p} b_{0}\right) \neq 0$, but

$$
\left(a_{0} b_{n}^{p}, a_{1} b_{n-1}^{p}, \ldots, a_{n} b_{0}^{p}\right)=\lambda\left(a_{0}, a_{1}, \ldots, a_{n}\right)
$$

for some nonzero constant $\lambda$.

Due to special forms of these vectors, we can summarize the above two cases as follows:

(i) $\left(a_{0} b_{n}, a_{1} b_{n-1}, \ldots, a_{n} b_{0}\right)=0$.

(ii) $\left(a_{0} b_{n}, a_{1} b_{n-1}, \ldots, a_{n} b_{0}\right) \neq 0$, but

$$
\left(a_{0} b_{n}^{p}, a_{1} b_{n-1}^{p}, \ldots, a_{n} b_{0}^{p}\right)=\lambda\left(a_{0}, a_{1}, \ldots, a_{n}\right)
$$

for some nonzero constant $\lambda$.

If the condition (i) holds for all $n \geq 0$, then either $\left(a_{n}\right) \equiv 0$ or $\left(b_{n}\right) \equiv 0$, which are acceptable (trivial) cases for equality. Hence, we assume that $\left(a_{n}\right) \not \equiv 0$ and $\left(b_{n}\right) \not \equiv 0$. Let $k \geq 0$ and $\ell \geq 0$ be the smallest integers such that $a_{k} \neq 0$ and $b_{\ell} \neq 0$. Therefore, (3.1) holds for $n=k+\ell$. Since in the vector $\left(a_{0} b_{k+\ell}^{p}, a_{1} b_{k+\ell-1}^{p}, \ldots, a_{k+\ell} b_{0}^{p}\right)$ there is just one nonzero component, i.e., $a_{k} b_{\ell}^{p}$, we deduce that besides $a_{0}=\cdots=a_{k-1}=0$ (assumption), we also must have $a_{k+1}=\cdots=a_{k+\ell}=0$. For other indices - and this is the second instance where an inequality should turn into an equality - note that to have equality in

$$
\left(\sum_{i=0}^{k+\ell} a_{i}\right)^{\frac{1}{q}}\left(\sum_{i=0}^{k+\ell} a_{i} b_{k+\ell-i}^{p}\right)^{\frac{1}{p}} \leq\left(\sum_{i=0}^{\infty} a_{i}\right)^{\frac{1}{q}}\left(\sum_{i=0}^{k+\ell} a_{i} b_{k+\ell-i}^{p}\right)^{\frac{1}{p}}
$$

we must have

$$
a_{k+\ell+1}=a_{k+\ell+2}=\cdots=0 .
$$


In short, $a_{n}=0$ for all values of $n \geq 0$ except possibly $n=k$. Conversely, it is easy to verify that for any such sequence,

$$
\left(\sum_{n=0}^{\infty}\left(\sum_{m=0}^{n} a_{m} b_{n-m}\right)^{p}\right)^{\frac{1}{p}}=a_{k}\left(\sum_{n=0}^{\infty} b_{n}^{p}\right)^{\frac{1}{p}}=\left(\sum_{n=0}^{\infty} a_{n}\right)\left(\sum_{n=0}^{\infty} b_{n}^{p}\right)^{\frac{1}{p}}
$$

for all choices of $\left(b_{n}\right)$.

\section{ACKNOWLEDGEMENT}

The author thanks the anonymous referee for helpful remarks and constructive suggestions.

\section{REFERENCES}

[1] Raymond Cheng and William T. Ross, An inner-outer factorization in $\ell^{p}$ with applications to ARMA processes, J. Math. Anal. Appl. 437 (2016), no. 1, 396-418, DOI 10.1016/j.jmaa.2016.01.009. MR.3451972

[2] G. H. Hardy, Divergent Series, Oxford, at the Clarendon Press, 1949. MR0030620

[3] Javad Mashreghi, Representation theorems in Hardy spaces, London Mathematical Society Student Texts, vol. 74, Cambridge University Press, Cambridge, 2009. MR2500010

Université Laval, Département de mathématiques et de statistique, Québec City, QuÉBec, G1V 0A6, CANADA

E-mail address: javad.mashreghi@mat.ulaval.ca 